\title{
Cardiac rehabilitation after myocardial infarction: a comparison between the standard and home-based cardiac rehabilitation programs
}

\begin{abstract}
Background: Cardiovascular disease (CVD) has emerged as the leading cause of death worldwide. Multiple meta-analyses have demonstrated that cardiac rehabilitation (CR) reduces mortality in patients with coronary artery disease. Despite guidelines recommending the use of CR programs for patients with ST segment elevation myocardial infarction (STEMI) participation in these programs continues to be low which had led to the development of alternative models of CR.
\end{abstract}

Objective: To evaluate the efficacy of home-based cardiac rehabilitation (HBCR) program in patients presenting with STEMI in a comparison with the standard inhospital CR program.

Methods: The study included 70 Patients referred for cardiac rehabilitation unit at Ain shams university hospitals after STEMI successfully treated by primary PCI.

Patients were subdivided into two (2) groups according to patients' preference to different modalities of cardiac rehabilitation:

a. Group (A): 35 patients who underwent regular in-hospital cardiac rehabilitation

b. Group (B): 35 patients who couldn't undergo regular in-hospital cardiac rehabilitation and preferred to undergo home-based cardiac rehabilitation

Outcome measures were assessed at baseline and after completion of the CR program in the form of:

a. $12 \mathrm{hr}$ fasting lipid profile (TC, HDL, LDL and TGs).

b. Transthoracic echocardiographic examination for assessment of Ejection fraction (EF\%) by 2D modified biplane Simpson's method

c. Symptom limited treadmill exercise stress test using Modified Bruce protocol: The following parameters were recorded: Resting blood pressure, resting heart rate (HR) and maximum achieved HR, HR recovery at 1 minute (HRR1), HR reserve and metabolic equivalents of task (METs) achieved.

Results: No significant differences between both groups regarding baseline lipid profile, $\mathrm{EF} \%$ and Exercise test parameters before starting the $\mathrm{CR}$ program. There was a statistically significant improvement in Lipid profile, EF\% and Exercise test parameters (METs, Exercise time, peak HR, HR reserve and HRR) after completion of the CR program in both groups with no statistically significant difference between both groups regarding delta changes in the studied parameters.

Conclusion: Home-based cardiac rehabilitation does not have inferior outcomes compared to hospital-based supervised program in post MI patients and may offer an alternative model of $\mathrm{CR}$ for individuals less able to access center-based cardiac rehabilitation.

Keywords: exercise, cardiac rehabilitation, home-based cardiac rehabilitation, stemi, myocardial infarction
Volume I2 Issue I - 2019

\author{
Hazem Khorshid,Adham Abdeltawab, \\ Mohamed Menshawy, Tarek Zaki \\ Department of cardiology, Ain Shams University Hospital, Egypt
}

Correspondence: Hazem M Khorshid, Department of cardiology, Ain Shams University Hospital, Cairo, Egypt, Tel 202 24821894,Email hazemkhorshid@yahoo.com

Received: September 17, 2018 | Published: January 08, 2019
Abbreviations: CVD, cardiovascular disease; CR, cardiac rehabilitation; STEMI, ST segment elevation myocardial infarction; PCI, percutaneous coronary intervention; TC, total cholesterol; HDL, high density lipoprotein; LDL, low density lipoprotein; TGs; triglycerides; $\mathrm{EF} \%$, ejection fraction; $\mathrm{HR}$, heart rate; $\mathrm{HRR}$, heart rate recovery; MET, metabolic equivalents of task

\section{Introduction}

Cardiovascular diseases (CVD) represent the leading cause of death in the world, accounting for $30 \%$ of global deaths. ${ }^{1,2}$ Hospitalbased cardiac rehabilitation (CR) is well-established in the effective management of patients with acute coronary syndrome (ACS). CR has been associated with improved survival, quality of life, functional status and cardiovascular risk profile as well as reduction in hospital re-admission. ${ }^{3} \mathrm{CR}$ is an evidence-based form of secondary prevention; where exercise-based CR is currently a class I indication in clinical practice guidelines, including those for ST segment elevation $\mathrm{MI}^{4,5}$ however; referral is suboptimal and participation rates are estimated at $10-30 \%{ }^{6}$ with lowest participation in the elderly, females, ethnic groups, and low-income populations. Barriers limiting participation in traditional CR programs include cost, transport diffculties, busy work schedules, lack of perceived need and low referral rates by the 
treating physicians. ${ }^{7,8}$ These challenges have led to the emergence of alternative models of CR including home-based cardiac rehabilitation (HBCR). ${ }^{9}$ Hence the main aim of this study was to evaluate the efficacy of home-based cardiac rehabilitation (HBCR) program in patients presenting with ST elevation myocardial infarction (STEMI) in a comparison with the standard in-hospital rehabilitation program.

\section{Materials and methods}

The study was conducted on 70 patients who presented to the coronary care unit (CCU) of Ain Shams university hospital, in the period between October 2017 and March 2018 with STEMI and underwent successful primary percutaneous coronary intervention (PCI). The patients were subdivided according to patients' preference into two (2) groups:

a. Group (A): 35 patients who underwent regular in-hospital CR.

b. Group (B): 35 patients who couldn't undergo regular in-hospital $\mathrm{CR}$ and preferred to undergo home-based cardiac rehabilitation (HBCR)

\section{Inclusion criteria}

The study included patients of both genders with age $<70$ years, who had a definite diagnosis of acute STEMI as per guidelines ${ }^{10}$ and underwent successful primary PCI and successfully revascularized with end result of TIMI III flow. ${ }^{11}$

\section{Exclusion criteria}

a. Patients with residual significant coronary artery stenotic lesions.

b. Patient with less Then TIMI III flow after 1ry PCI.

c. Patient with ejection fraction (EF) less than $35 \%$.

d. Patients with decompensated Heart failure (HF).

e. Patients with acute myocarditis, pericarditis and endocarditis.

f. Significant valvular Heart Disease.

g. Patients who had debilitating disease preventing them from attending follow-ups (Chronic renal failure, liver cell failure ... etc).

h. Patients with cardiovascular contraindication to exercise (e.g. severe aortic stenosis, hypertrophic obstructive cardiomyopathy, significant arrhythmias or heart block).

i. Patient with neuro-muscular disability interfering with exercise.

j. Patients with implanted CRT or ICD.

k. Patients with cognitive impairment or severe mental illness.

1. Patient refusal.

\section{Initial patients' assessment}

All patients were subjected to baseline history taking; clinical examination and 12 lead resting ECG.

\section{Outcome measures were assessed at baseline and after completion of the CR program}

1. $12 \mathrm{hr}$ fasting lipid profile: Total Cholesterol (TC), triglycerides (TG), high density lipoprotein cholesterol (HDL-C), and low density lipoprotein cholesterol (LDL-C)

2. Trans-thoracic echocardiography: Transthoracic echocardiographic examination with machine-integrated ECG recording was performed, using Vivid E9 machine with an M4S matrix sector array probe with a frequency of 2.5 Mega $\mathrm{Hz}$ (General Electric Vingmed Ultrasound, Horten, Norway). Ejection fraction $(\mathrm{EF} \%)$ was assessed by $2 \mathrm{D}$ modified biplane Simpson's method.

3. Symptom limited treadmill exercise stress test using Modified Bruce protocol: The following parameters were recorded: Resting blood pressure (BP), resting heart rate (HR) and maximum achieved HR, HR recovery at 1 minute (HRR1), HR reserve and metabolic equivalents of task (METs) achieved.

\section{Exercise based cardiac rehabilitation protocol \\ Group A}

The patients in group A joined the standard CR program in the CR unit in our institute. Moderate intensity exercise training twice weekly for 3 months was prescribed targeting exercise heart rate of $40-60 \%$ of HR reserve calculated from preliminary symptom limited exercise stress test by modified Bruce protocol. The duration of each session was 30-45 minutes. The progress of exercise intensity was monitored by continuous ECG recording, monitoring of heart rate, blood pressure and symptoms as well as the rate of perceived exertion (RPE) on Borg scale where the patients were exercised at an RPE of 11-13 in the absence of symptoms. Only the patients who completed the CR program, attending $>80 \%$ of the sessions were included in the study.

\section{Group B:}

Included patients who couldn't undergo regular in-hospital cardiac rehabilitation and preferred to undergo HBCR. The patients received group education sessions regarding home based exercise program, warning symptoms and were given a hotline phone number to call in case of emergencies. All Patients in group B underwent a model of HBCR based on continuous walking training, 3-5 times per week for 3 months. The duration of each session was 30-40 minutes including 5-10 minutes of warming up, 20-30 minutes of moderate intensity exercise, and 5-10 minutes of cooling down. The patients were instructed to maintain moderate exercise intensity (corresponding to RPE of 11-13 on Borg scale) and to monitor their HR during exercise if possible (using finger tip pulse oximeter that also gives HR readings or treadmills that have hand-grip HR sensors) to maintain $40-60 \%$ of HR reserve calculated from a preliminary symptom limited exercise stress test by modified Bruce protocol. To control their adherence to exercise, the patients were instructed to document their exercise in an exercise monitoring chart. The patients were followed up by a telephone call from the CR physician twice weekly and were informed to contact him if they had any abnormal symptoms during their exercise (chest pain, dysnea or fatigue). The patients were followed up clinically every 2 weeks in our CR unit.

The study was approved by the Ethics committee of our institute. And informed consent was obtained from the patients ensuring privacy of their data.

\section{Statistical analysis}

Data were collected, revised, coded and entered to the Statistical Package for Social Science (IBM SPSS) version 23. The quantitative data were presented as mean, standard deviations and ranges when their distribution found parametric while qualitative data were presented as number and percentages. The comparison between two independent 
groups with qualitative data was done by using Chi-square test. The comparison between two independent groups with quantitative data and parametric distribution was done by using Independent t-test. The $\mathrm{p}$-value was considered significant as the following:

$$
\begin{aligned}
& \mathrm{p}>0.05 \text { : Non significant (NS) } \\
& \mathrm{p}<0.05 \text { : Significant (S) } \\
& \mathrm{p}<0.001 \text { : Highly significant (HS). }
\end{aligned}
$$

\section{Results}

\section{Baseline demographic data and clinical risk factors}

1. The mean age of the patients in group A and B was $46.97 \pm 9.2$ years and $47.94 \pm 10.05$ years respectively with no significant difference between the 2 groups $(\mathrm{P}-\mathrm{value}=0.675)($ Table 1$)$.

2. Group A included $30(85.7 \%)$ males and $5(14.3 \%)$ females while Group B included 32 (91.4\%) males and 3 (8.6\%) females with no significant difference between the 2 groups $(\mathrm{P}-\mathrm{value}=0.452)$ (Table 1).
3. Regarding modifiable risk factors in group (A): $28(80.0 \%)$ were hypertensive, $20(57.1 \%)$ were diabetics and $24(68.6 \%)$ were smokers, while in group (B): $23(65.7 \%)$ were hypertensive, 22 $(62.9 \%)$ were diabetics and $28(80.0 \%)$ were smokers, with no significant difference between the 2 groups $(\mathrm{p}<0.05)$ (Table 1).

\section{Comparison between the 2 studied groups regarding the baseline studied outcome parameters:}

1. The mean EF\% was $48.26 \pm 8.34 \%$ in Group A and $46.11 \pm 8.70 \%$ in Group B with no statistically significant difference between the 2 groups $(\mathrm{p}=0.296)$ (Table 2$)$.

2. There were no significant differences between the 2 studied groups regarding baseline lipid profile measurements (TC, LDL-C, HDL-C and TG) (Table 3) and baseline exercise test parameters (Functional capacity expressed in "METs", exercise time, resting heart rate, maximum heart rate, heart rate reserve, heart rate recovery and arterial blood pressure) before enrolment into the $\mathrm{CR}$ program (Table 2).

Table I Baseline demographic data and clinical risk factors

\begin{tabular}{lllllll}
\hline \multirow{2}{*}{ Risk Factors } & & Group A & Group B & Test value & P-value & Sig. \\
\cline { 3 - 7 } & & $\mathbf{N = 3 5}$ & $\mathbf{N = 3 5}$ & & & \\
\hline \multirow{2}{*}{ Age (years) } & Mean \pm SD & $46.97 \pm 9.22$ & $47.94 \pm 10.05$ & $0.421^{*}$ & 0.675 & NS \\
& Range & $27-60$ & $30-66$ & & & \\
\multirow{2}{*}{ Gender } & Male & $30(85.7 \%)$ & $32(91.4 \%)$ & $0.565 \bullet$ & 0.452 & NS \\
Smoking & Female & $5(14.3 \%)$ & $3(8.6 \%)$ & & & \\
HTN & & $24(68.6 \%)$ & $28(80.0 \%)$ & $1.197 \bullet$ & 0.274 & NS \\
DM & & $28(80.0 \%)$ & $23(65.7 \%)$ & $1.806 \bullet$ & 0.179 & NS \\
\hline
\end{tabular}

\begin{tabular}{|c|c|c|c|c|c|c|}
\hline \multirow{2}{*}{ Before } & & \multirow{2}{*}{$\begin{array}{l}\text { Group A } \\
(\mathrm{N}=35)\end{array}$} & \multirow{2}{*}{$\begin{array}{l}\text { Group B } \\
(\mathrm{N}=35)\end{array}$} & \multirow[t]{2}{*}{ Test value } & \multirow[t]{2}{*}{ P-value } & \multirow[t]{2}{*}{ Sig. } \\
\hline & & & & & & \\
\hline \multirow{2}{*}{ METs } & Mean \pm SD & $11.63 \pm 2.12$ & $10.64 \pm 2.56$ & \multirow{2}{*}{-1.756} & \multirow{2}{*}{0.084} & \multirow{2}{*}{ NS } \\
\hline & Range & $8-17$ & $4-15$ & & & \\
\hline \multirow{2}{*}{ Exercise time(min) } & Mean \pm SD & $13.93 \pm 2.12$ & $13.10 \pm 2.91$ & \multirow{2}{*}{-1.361} & \multirow{2}{*}{0.178} & \multirow{2}{*}{ NS } \\
\hline & Range & $10.33-19.33$ & $5.51-18.2$ & & & \\
\hline \multirow{2}{*}{ Resting HR (bpm) } & Mean \pm SD & $70.94 \pm 5.66$ & $74.34 \pm 9.66$ & \multirow{2}{*}{1.797} & \multirow{2}{*}{0.077} & \multirow{2}{*}{ NS } \\
\hline & Range & $58-82$ & $55-5$ & & & \\
\hline \multirow{2}{*}{ Peak HR (bpm) } & Mean \pm SD & $136.77 \pm 12.36$ & $131.60 \pm 11.06$ & \multirow{2}{*}{-1.844} & \multirow{2}{*}{0.069} & \multirow{2}{*}{ NS } \\
\hline & Range & $115-166$ & $100-152$ & & & \\
\hline \multirow{2}{*}{ HR reserve (bpm) } & Mean \pm SD & $65.66 \pm 13.02$ & $61.09 \pm 9.17$ & \multirow{2}{*}{-1.699} & \multirow{2}{*}{0.094} & \multirow{2}{*}{ NS } \\
\hline & Range & $38-94$ & $40-78$ & & & \\
\hline \multirow{2}{*}{ HR after 1 min (beat) } & Mean \pm SD & $118.20 \pm 11.85$ & $114.26 \pm 9.72$ & \multirow{2}{*}{-1.522} & \multirow{2}{*}{0.133} & \multirow{2}{*}{ NS } \\
\hline & Range & $90-150$ & $100-130$ & & & \\
\hline \multirow{2}{*}{ HRR (bpm) } & Mean \pm SD & $16.03 \pm 13.71$ & $19.57 \pm 8.70$ & \multirow{2}{*}{1.291} & \multirow{2}{*}{0.201} & \multirow{2}{*}{ NS } \\
\hline & Range & $0-76$ & $7-36$ & & & \\
\hline \multirow{2}{*}{ rSBP (mmhg) } & Mean \pm SD & $116.29 \pm 15.40$ & $118.71 \pm 12.91$ & 0.71 & 0.4 & NS \\
\hline & Range & $90-150$ & $85-150$ & 0.715 & $0.4 / 1$ & NS \\
\hline rDBP (mmhg) & Mean \pm SD & $75.14 \pm 10.67$ & $70.29 \pm 10.14$ & -1.952 & 0.055 & NS \\
\hline & Range & $60-100$ & $50-90$ & & 0.053 & \\
\hline $\mathrm{EF} \%$ & Mean \pm SD & $48.26 \pm 8.34$ & $46.11 \pm 8.70$ & -1052 & 0296 & NS \\
\hline & Range & $36-61$ & $35-66$ & & 0.230 & $10 \mathrm{~s}$ \\
\hline
\end{tabular}

HTN, hypertension; DM, diabetes mellitus

Table 2 Comparison between the 2 studied groups regarding the baseline Exercise test and EF\%

MET, Metabolic equivalent of task; HR, Heart rate; HRR, Heart rate recovery; rSBP, resting systolic blood pressure; rDBP, resting diastolic blood pressure; EF, Ejection fraction 


\section{Impact of hospital based CR on group A}

There was a statistically significant improvement in exercise test parameters after completion of the CR program. Functional capacity expressed in "METs" significantly increased from $11.63 \pm 2.12$ to
$12.71 \pm 2.11(\mathrm{p}<0.001)$. Exercise time, maximum HR, HR reserve and HRR1 also showed statistically significant increase $(p<0.001)$. Resting heart rate was significantly decreased from $70.94 \pm 5.66 \mathrm{bpm}$ to $66.17 \pm 5.66 \mathrm{bpm}(\mathrm{p}<0.001)$. There was no significant change in the resting $\mathrm{BP}(\mathrm{p}>0.05)$ (Table 4$)$.

Table 3 Comparison between the two studied groups regarding baseline lipid profile before CR

\begin{tabular}{|c|c|c|c|c|c|c|}
\hline Before & & Group A $(N=35)$ & Group $B(N=35)$ & Test value & P-value & Sig. \\
\hline \multirow{2}{*}{ T.Chol (mg/dL) } & Mean \pm SD & $170.69 \pm 30.34$ & $163.89 \pm 27.66$ & \multirow{2}{*}{-0.98} & \multirow{2}{*}{0.331} & \multirow{2}{*}{ NS } \\
\hline & Range & $122-235$ & $111-235$ & & & \\
\hline \multirow{2}{*}{ LDL (mg/dL) } & Mean \pm SD & $98.23 \pm 25.53$ & $92.66 \pm 24.59$ & \multirow{2}{*}{-0.93} & \multirow{2}{*}{0.356} & \multirow{2}{*}{ NS } \\
\hline & Range & $61-150$ & $51-153$ & & & \\
\hline \multirow{2}{*}{ HDL (mg/dL) } & Mean \pm SD & $43.66 \pm 8.75$ & $41.46 \pm 9.94$ & \multirow{2}{*}{-0.985} & \multirow{2}{*}{0.328} & \multirow{2}{*}{ NS } \\
\hline & Range & $31-57$ & $25-62$ & & & \\
\hline \multirow{2}{*}{ TG (mg/dL) } & Mean \pm SD & $137.26 \pm 49.97$ & $129.20 \pm 46.09$ & \multirow{2}{*}{-0.701} & \multirow{2}{*}{0.486} & \multirow{2}{*}{ NS } \\
\hline & Range & $82-311$ & $77-327$ & & & \\
\hline
\end{tabular}

T Chol, total cholesterol; LDL, low density lipoprotein; HDL, high density lipoprotein; TG, triglycerides

Table 4 Comparison regarding Exercise test parameters, EF\% and lipid profile before and after CR in group A

\begin{tabular}{|c|c|c|c|c|c|c|}
\hline \multirow{2}{*}{ Group A } & & \multirow{2}{*}{ Before CR } & \multirow{2}{*}{ After CR } & \multicolumn{3}{|c|}{ Paired t-test } \\
\hline & & & & $\mathbf{t}$ & p-value & Sig. \\
\hline \multirow{2}{*}{ METs } & Mean \pm SD & $11.63 \pm 2.12$ & $12.71 \pm 2.11$ & \multirow{2}{*}{-14.423} & \multirow{2}{*}{$<0.001$} & \multirow{2}{*}{ HS } \\
\hline & Range & $8-17$ & $9-18$ & & & \\
\hline \multirow{2}{*}{ Exercise time(min) } & Mean \pm SD & $13.93 \pm 2.12$ & $15.24 \pm 2.50$ & \multirow{2}{*}{-5.066} & \multirow{2}{*}{$<0.001$} & \multirow{2}{*}{ HS } \\
\hline & Range & $10.33-19.33$ & $10.6-19.08$ & & & \\
\hline \multirow{2}{*}{ Resting HR (bpm) } & Mean \pm SD & $70.94 \pm 5.66$ & $66.17 \pm 5.66$ & \multirow{2}{*}{5.236} & \multirow{2}{*}{$<0.001$} & \multirow{2}{*}{ HS } \\
\hline & Range & $58-82$ & $56-77$ & & & \\
\hline \multirow{2}{*}{ Peak HR (bpm) } & Mean \pm SD & $136.77 \pm 12.36$ & & \multirow{2}{*}{-9.646} & \multirow{2}{*}{$<0.001$} & \multirow{2}{*}{ HS } \\
\hline & Range & $115-166$ & $124-167$ & & & \\
\hline \multirow{2}{*}{ HR reserve (bpm) } & Mean \pm SD & $65.66 \pm 13.02$ & $73.29 \pm 13.74$ & \multirow{2}{*}{-8.973} & \multirow{2}{*}{$<0.001$} & \multirow{2}{*}{ HS } \\
\hline & Range & $38-94$ & $43-99$ & & & \\
\hline \multirow{2}{*}{ HR after 1 min (beat) } & Mean \pm SD & $118.20 \pm 11.85$ & $107.97 \pm 15.71$ & \multirow{2}{*}{5.95} & \multirow{2}{*}{$<0.001$} & \multirow{2}{*}{ HS } \\
\hline & Range & $90-150$ & $73-145$ & & & \\
\hline \multirow{2}{*}{ HRR(bpm) } & Mean \pm SD & $16.03 \pm 13.71$ & $22.77 \pm 13.61$ & \multirow{2}{*}{-8.915} & \multirow{2}{*}{$<0.001$} & HS \\
\hline & Range & $0-76$ & $7-79$ & & & חIS \\
\hline CDD (mmb & Mean \pm SD & $116.29 \pm 15.40$ & $116.00 \pm 9.73$ & 0 & 1 & NST \\
\hline IDDF (IIIIII) & Range & $90-150$ & $100-130$ & 0 & 1 & IVS \\
\hline $\mathrm{rDRP}(\mathrm{mmhr})$ & Mean \pm SD & $75.14 \pm 10.67$ & $72.86 \pm 8.25$ & 1604 & 0118 & NS \\
\hline & Range & $60-100$ & $60-90$ & & & \\
\hline$F[0 /$ & Mean \pm SD & $48.26 \pm 8.34$ & $52.11 \pm 8.22$ & 8154 & $-0 \Omega 01$ & HS \\
\hline & Range & $36-61$ & $36-64$ & & & \\
\hline T $\mathrm{Ch}_{\mathrm{b}} 1$ (m) & Mean \pm SD & $170.69 \pm 30.34$ & $138.23 \pm 23.80$ & 056 & 001 & WS \\
\hline 1.CHOI (IIIg/ (UL) & Range & $122-235$ & $96-200$ & 8.056 & $<0.001$ & $\mathrm{HS}$ \\
\hline I DI (mo/dI) & Mean \pm SD & $98.23 \pm 25.53$ & $73.14 \pm 15.36$ & 7508 & $<0 \Omega 01$ & $\mathrm{HS}$ \\
\hline LDL (mg/dL) & Range & $61-150$ & $45-120$ & 1.500 & -0.001 & HS \\
\hline HDI $(\mathrm{moldI})$ & Mean \pm SD & $43.66 \pm 8.75$ & $53.74 \pm 9.78$ & 068 & 01 & USC \\
\hline HDL (IIIg/CL) & Range & $31-57$ & $33-72$ & -9.00 & -0.001 & ПIS \\
\hline & Mean \pm SD & $137.26 \pm 49.97$ & $119.83 \pm 26.44$ & 13 & 0 & $\mathrm{~S}$ \\
\hline 10 (III)/ UL) & Range & $82-311$ & $48-164$ & 2.110 & & \\
\hline
\end{tabular}

MET, metabolic equivalent of task, HR, heart rate; HRR, heart rate recovery; rSBP, resting systolic blood pressure; rDBP, resting diastolic blood pressure; EF, ejection fraction; T Chol, total cholesterol; DL, low density lipoprotein; HDL, High density lipoprotein;TG, triglycerides 
There was a statistically significant improvement in LV systolic function after completion of the $\mathrm{CR}$ program as $\mathrm{EF} \%$ increased from $48.26 \pm 8.34 \%$ to $52.11 \pm 8.22 \%(\mathrm{p}<0.001)$ (Table 4$)$. There was also a statistically highly significant improvement in lipid profile measurements after completion of the CR program in group A as total cholesterol, LDL-C and TG levels were significantly decreased $(170.69 \pm 30.34 \mathrm{mg} / \mathrm{dl}$ vs $138.23 \pm 23.80 \mathrm{mg} / \mathrm{dl}, 98.23 \pm 25.53 \mathrm{mg} / \mathrm{dl}$ vs $73.14 \pm 15.36 \mathrm{mg} / \mathrm{dl}$ and $137.26 \pm 49.97 \mathrm{mg} / \mathrm{dl}$ vs $119.83 \pm 26.44 \mathrm{mg} / \mathrm{dl}$ respectively) $(\mathrm{p}<0.001)$. On the other hand HDL-C was significantly increased $(43.66 \pm 8.75 \mathrm{mg} / \mathrm{dl}$ vs $53.74 \pm 9.78 \mathrm{mg} / \mathrm{dl})(\mathrm{p}<0.001)$ (Table $4)$.

\section{Impact of home-based CR on group B}

There was a statistically significant improvement in Exercise test parameters after completion of the HBCR program. Functional capacity expressed in "METs" significantly increased from $10.64 \pm 2.56$ to $11.94 \pm 2.44(p<0.001)$. Exercise time, maximum HR, HR reserve and HRR1 also showed statistically significant increase $(\mathrm{p}<0.001)$. Resting HR was significantly decreased from $74.34 \pm 9.66 \mathrm{bpm}$ to $68.60 \pm 8.06 \mathrm{bpm}(\mathrm{p}<0.001)$. There was no significant change in the resting BP $(\mathrm{p}>0.05)$ (Table 5). There was a statistically significant improvement in LV systolic function at the end of HBCR program as $\mathrm{EF} \%$ increased from $46.11 \pm 8.70 \%$ to $50.37 \pm 8.12 \%$ ( $<<0.001$ ) (Table $5)$. There was a statistically significant improvement in lipid profile measurements after HBCR program in group B as TC, LDL-C and TG were significantly decreased $(163.89 \pm 27.66 \mathrm{mg} / \mathrm{dl}$ vs $122.80 \pm 20.64$ $\mathrm{mg} / \mathrm{dl}, 92.66 \pm 24.59 \mathrm{mg} / \mathrm{dl}$ vs $67.74 \pm 16.91 \mathrm{mg} / \mathrm{dl}$ and $129.20 \pm 46.09$ $\mathrm{mg} / \mathrm{dl}$ vs $104.54 \pm 29.26 \mathrm{mg} / \mathrm{dl}$ respectively) ( $<0.001)$. HDL-C was significantly increased $(41.46 \pm 9.94 \mathrm{mg} / \mathrm{dl}$ vs $52.66 \pm 10.46 \mathrm{mg} / \mathrm{dl})$ $(\mathrm{p}<0.001)$ (Table 5).

Table 5 Comparison regarding Exercise test parameters, EF\% and lipid profile before and after HBCR in group B

\begin{tabular}{|c|c|c|c|c|c|c|}
\hline \multirow{2}{*}{ Group B } & & \multirow{2}{*}{ Before HBCR } & \multirow{2}{*}{ After HBCR } & \multicolumn{3}{|c|}{ Paired t-test } \\
\hline & & & & t & p-value & Sig. \\
\hline \multirow{2}{*}{ METs } & Mean \pm SD & $10.64 \pm 2.56$ & $11.94 \pm 2.44$ & \multirow{2}{*}{-10.261} & \multirow{2}{*}{$<0.001$} & \multirow{2}{*}{ HS } \\
\hline & Range & $4-15$ & $5-16$ & & & \\
\hline \multirow{2}{*}{ Exercise time (min) } & Mean \pm SD & $13.10 \pm 2.91$ & $14.51 \pm 2.91$ & \multirow{2}{*}{-4.735} & \multirow{2}{*}{$<0.001$} & \multirow{2}{*}{ HS } \\
\hline & Range & $5.51-18.2$ & $8.45-19.24$ & & & \\
\hline \multirow{2}{*}{ Resting HR (bpm) } & Mean \pm SD & $74.34 \pm 9.66$ & $68.60 \pm 8.06$ & \multirow{2}{*}{6.425} & \multirow{2}{*}{$<0.001$} & \multirow{2}{*}{ HS } \\
\hline & Range & $55-5$ & $53-85$ & & & \\
\hline \multirow{2}{*}{ Peak HR (bpm) } & Mean \pm SD & $131.60 \pm 11.06$ & $137.77 \pm 10.99$ & \multirow{2}{*}{-5.608} & \multirow{2}{*}{$<0.001$} & \multirow{2}{*}{ HS } \\
\hline & Range & $100-152$ & $105-158$ & & & \\
\hline \multirow{2}{*}{ HR reserve (bpm) } & Mean \pm SD & $61.09 \pm 9.17$ & $67.77 \pm 9.12$ & \multirow{2}{*}{-8.504} & \multirow{2}{*}{$<0.001$} & \multirow{2}{*}{ HS } \\
\hline & Range & $40-78$ & $47-85$ & & & \\
\hline \multirow{2}{*}{ HR after 1 min (beat) } & Mean \pm SD & $114.26 \pm 9.72$ & $104.77 \pm 15.24$ & \multirow{2}{*}{4.361} & \multirow{2}{*}{$<0.001$} & \multirow{2}{*}{ HS } \\
\hline & Range & $100-130$ & $70-145$ & & & \\
\hline \multirow{2}{*}{ HRR (bpm) } & Mean \pm SD & $19.57 \pm 8.70$ & $27.97 \pm 18.49$ & \multirow{2}{*}{-2.358} & \multirow{2}{*}{0.024} & $\mathrm{~S}$ \\
\hline & Range & $7-36$ & $10-82$ & & & $\mathrm{~S}$ \\
\hline $\mathrm{rSBP}(\mathrm{mmho})$ & Mean \pm SD & $118.71 \pm 12.91$ & $116.00 \pm 10.63$ & 1127 & 027 & NS \\
\hline IDDT (IIIIII) & Range & $85-150$ & $100-130$ & 1.122 & 0.21 & Nis \\
\hline $\mathrm{rDRP}(\mathrm{mmhs})$ & Mean \pm SD & $70.29 \pm 10.14$ & $69.29 \pm 7.29$ & 0631 & 0.532 & NS \\
\hline 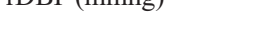 & Range & $50-90$ & $60-80$ & 0.051 & 0.032 & NOS \\
\hline FF\% & Mean \pm SD & $46.11 \pm 8.70$ & $50.37 \pm 8.12$ & 5171 & $<0001$ & HS \\
\hline 5170 & Range & $35-66$ & $38-66$ & $-11 / 1$ & -0.001 & III \\
\hline $\mathrm{T} C \mathrm{Cb} / \mathrm{mo} / \mathrm{dI})$ & Mean \pm SD & $163.89 \pm 27.66$ & $122.80 \pm 20.64$ & 11163 & $<0.001$ & HS \\
\hline 1.01100 (1119/(L) & Range & $111-235$ & $80-170$ & 11.105 & -0.001 & \\
\hline JDI (maldI) & Mean \pm SD & $92.66 \pm 24.59$ & $67.74 \pm 16.91$ & 0018 & $<0001$ & HS \\
\hline LDL (III)/UL) & Range & $51-153$ & $40-113$ & 9.010 & -0.001 & П10 \\
\hline HDI (maldi) & Mean \pm SD & $41.46 \pm 9.94$ & $52.66 \pm 10.46$ & 8614 & 01 & HS \\
\hline (IIIS/U) & Range & $25-62$ & $33-72$ & -0.017 & -0.001 & Hiv \\
\hline $\mathrm{TG} / \mathrm{mo} / \mathrm{dI}$ & Mean \pm SD & $129.20 \pm 46.09$ & $104.54 \pm 29.26$ & 3705 & 00. & US \\
\hline $10(\mathrm{~min} / \mathrm{dL})$ & Range & $77-327$ & $48-160$ & 3.105 & 0.001 & HS \\
\hline
\end{tabular}

MET, metabolic equivalent of task, HR, heart rate; HRR, heart rate recovery; rSBP, resting systolic blood pressure; rDBP, resting diastolic blood pressure; EF, ejection fraction;T Chol, total cholesterol; DL, low density lipoprotein; HDL, High density lipoprotein;TG, triglycerides 


\section{Comparing the impact of $\mathrm{CR}$ on the studied parameters in the 2 studied groups}

There was no statistically significant difference between the 2 studied groups regarding changes in exercise test parameters, $\mathrm{EF} \%$ or lipid profile after completion of the CR programs (Table 6).

\section{Discussion}

\section{Standard CR}

In the present study Group A (Post MI patients who completed standard exercise hospital based-CR program) showed significant improvement in exercise parameters (METs, Exercise time, peak HR, HR reserve and HRR1), LV systolic function (EF\%) and lipid profile after completion of the standard CR. The positive impact of regular physical activity has been well documented in several studies. ${ }^{12-14}$ The role of exercise-based CR is well established in the secondary prevention of coronary artery disease (CAD). Beneficial cardioprotective effects of exercise training include improvements in atherosclerotic CVD risk profile as well as favourable antiatherogenic, anti-inflammatory, and antithrombotic effects. Exercise training also has antiarrhythmic and anti-ischemic effects with improved myocardial perfusion, raised ischemic threshold and ischemic preconditioning of the myocardium. ${ }^{15-18}$

Table 6 Comparison between the delta change in Exercise test parameters, EF\% and lipid profile after completion of CR in the 2 studied groups

\begin{tabular}{lllllll}
\hline \multirow{2}{*}{ Difference } & & Group A & Group B & Test value & P-value & Sig. \\
\cline { 2 - 4 } & & $\mathbf{( N = 3 5 )}$ & $\mathbf{( N = 3 5 )}$ & & & \\
\hline$\Delta$ METS & Mean \pm SD & $1.08 \pm 0.45$ & $1.30 \pm 0.75$ & 1.454 & 0.151 & NS \\
$\Delta$ Exercise time & Mean \pm SD & $1.31 \pm 1.53$ & $1.41 \pm 1.76$ & 0.251 & 0.802 & NS \\
$\Delta$ Resting HR & Mean \pm SD & $-4.77 \pm 5.39$ & $-5.74 \pm 5.29$ & -0.761 & 0.449 & NS \\
$\Delta$ Peak HR & Mean \pm SD & $6.26 \pm 3.84$ & $6.17 \pm 6.51$ & -0.067 & 0.947 & NS \\
$\Delta$ HR reserve & Mean \pm SD & $7.63 \pm 5.03$ & $6.69 \pm 4.65$ & -0.814 & 0.418 & NS \\
$\Delta$ HR after 1 min & Mean \pm SD & $-10.23 \pm 10.17$ & $-9.49 \pm 12.87$ & 0.268 & 0.79 & NS \\
$\Delta$ HRR & Mean \pm SD & $6.74 \pm 4.47$ & $8.40 \pm 21.08$ & 0.455 & 0.651 & NS \\
$\Delta$ Systolic BP & Mean \pm SD & $-0.29 \pm 13.12$ & $-2.71 \pm 14.31$ & -0.827 & 0.411 & NS \\
$\Delta$ Diastolic BP & Mean \pm SD & $-2.29 \pm 8.43$ & $-1.00 \pm 9.38$ & 0.603 & 0.548 & NS \\
$\Delta$ EF & Mean \pm SD & $3.86 \pm 2.80$ & $4.26 \pm 4.87$ & 0.421 & 0.675 & NS \\
$\Delta$ T. Chol & Mean \pm SD & $-32.46 \pm 23.83$ & $-41.09 \pm 21.77$ & -1.581 & 0.118 & NS \\
$\Delta$ LDL & Mean \pm SD & $-25.09 \pm 19.77$ & $-24.91 \pm 16.34$ & 0.040 & 0.969 & NS \\
$\Delta$ HDL & Mean $\pm S D$ & $10.07 \pm 6.16$ & $11.20 \pm 7.69$ & 0.676 & 0.501 & NS \\
$\Delta$ TG & Mean $\pm S D$ & $-17.43 \pm 38.00$ & $-24.66 \pm 39.37$ & -0.782 & 0.437 & NS \\
\hline
\end{tabular}

MET, metabolic equivalent of task, HR, heart rate; HRR, heart rate recovery; rSBP, resting systolic blood pressure; rDBP, resting diastolic blood pressure; EF, ejection fraction; T Chol, total cholesterol; DL, low density lipoprotein; HDL, High density lipoprotein; TG, triglycerides

\section{Effect of CR on lipid profile}

The improvement in lipid profile in group A after completion of the CR program is concordant with the results of the a meta-analysis that demonstrated the benefits of participation in $\mathrm{CR}$ and showed significant reduction in TC, LDL-C and TG with CR, with a trend towards increase in HDL-C. ${ }^{19}$ Significant reduction in BP was also reported after CR..$^{20,21}$ Absence of significant change in resting BP after CR in the present study may be explained by the relatively small number of patients included and that the mean BP of both groups was controlled before enrolment in the study. In a study by El Demerdash et al CR not only improved the ischemic burden in patients with ischemic heart disease who are not suitable for revascularization but was also associated with statistically significant reduction in the incidence of smoking, significant reduction in the resting BP, LDL cholesterol level and HbA1c, while the HDL cholesterol level significantly increased. ${ }^{22}$

\section{Effect of CR on exercise test parameters}

The improvement in the exercise test parameters after completion of the CR program in group $\mathrm{A}$ is concordant with results of randomized, controlled trials (RCTs) which demonstrated that exercise training improves exercise endurance and walking distance by $10-25 \% .^{23,24}$ Also, a subanalysis of 2 RCTs showed that CR following MI resulted in significant increase in the physical activity levels. ${ }^{25}$ The increase in HRR post CR in the present study is supported by a systematic review by Snoek et al which included 8 studies and showed that regular exercise training increases HRR in patients with established heart disease. ${ }^{26}$

\section{Effect of CR on LV systolic function}

In concordance with our results, Haddadzadeh et al. ${ }^{27}$ reported that a12-week structured exercise training resulted in significant improvement of LVEF in post-event CAD patients. ${ }^{27}$

\section{Home based CR (HBCR)}

In the present study Group B (Post MI patients who underwent HBCR) showed significant improvement of exercise parameters, BP, LV Systolic function (EF\%) and lipid profile after completion of the standard CR with no significant difference as compared to group A. The results of the present study are supported by the Cochrane systematic review that showed that HBCR and centre-based CR provide similar benefits in patients with heart disease. The authors 
analysed 17 studies of 2172 patients and found no difference between HBCR and centre-based CR in terms of mortality, acute coronary events and functional capacity as well as significant improvement in TC, TG, LDL and systolic blood pressure. ${ }^{28}$

\section{Effect of HBCR on lipid profile}

The improvement in CV risk profile in the present study is concordant with Senuzun et al. ${ }^{29}$ who found that 12 -week home based exercise program resulted in significant improvement of total cholesterol $(\mathrm{P}=0.004)$, triglycerides $(\mathrm{P}=0.048)$, high-density lipoprotein-cholesterol $(\mathrm{P}=0.001)$ and low-density lipoproteincholesterol $(\mathrm{P}=0.039)$ as compared to the control group in patients with coronary artery disease. ${ }^{29}$ Similar results were demonstrated more recently in 2016 by Chen et al. ${ }^{30}$ who reported positive impact on modifiable risk-factors (including LDL, HDL) in patients with coronary artery disease attending HBCR program. ${ }^{30}$

\section{Effect of HBCR on exercise test parameters}

The improvement in the exercise parameters after HBCR in the present study is supported by previous studies. ${ }^{29-33}$ In a study by Mosleh et al. ${ }^{31}$ who compared community based cardiac rehabilitation with hospital based CR, found no significant differences regarding frequency or intensity of exercise immediately after the CR program or after 6 months in patients after acute myocardial infarction or post coronary revascularization. ${ }^{31}$ Our results are also concordant with a more recent RCT which studied the effectiveness and safety of HBCR in patients with stable CAD and showed no significant differences between the traditional cardiac rehabilitation group and the home based group regarding exercise time and METS achieved during the exercise test as well as the HR recovery in the first minute (which improved in both groups). ${ }^{33}$

Similar results were reported in post CABG patients by Shagufta et $\mathrm{al}^{34}$ who found that HBCR showed significant improvements in HRR and was equally effective as the supervised hospital-group in patients after CABG surgery. ${ }^{34}$ The improved exercise time and HRR is consistent with autonomic improvement and is associated with better prognosis and lower mortality in patients with coronary heart disease. ${ }^{35}$

\section{Effect of HBCR on LV systolic function}

The improvement in $\mathrm{EF} \%$ in both groups in the present study is supported by previous studies. Haddadzadeh et al. ${ }^{27}$ reported a significant increase in LVEF (46.9\% to 61.5\%) in post ACS patients who underwent a 12-week exercise program (either in the form of center-based or home-based) as compared to the control group (47.9\% to $47.6 \%)(\mathrm{P}=0.001)$ with no significant difference between the homebased and center-based groups. ${ }^{27} \mathrm{Also}$ in concordance with the present study, a recent study showed that early HBCR in post MI patients was associated with significant improvements in LVEF as well as global longitudinal strain, global radial strain and global circumferential strain (using 3-dimensional speckle tracking echocardiography) as compared to the control group at the 4-week follow-up. ${ }^{36}$

\section{Limitation}

The present study was a single center study which included a relatively small number of patients with a short period of follow up. Another limitation of the study was the difficulty to randomize the patients to hospital-based or home-based groups due to the universal barriers of $\mathrm{CR}$, which include accessibility, transport difficulties, cost and patients' preference. These limitations can be addressed in future studies.

\section{Conclusion}

The present study showed that HBCR does not have inferior outcomes compared to standard hospital-based supervised program in post MI patients. Based on the results of the present study, homebased programs may offer an alternative model of CR for individuals less able to access hospital-based CR. Choices should reflect patients' preferences, risk profile, insurance coverage and accessibility to the rehabilitation service.

\section{Acknowledgments}

None.

\section{Conflicts of interest}

No financial interest or any conflict of interest exists.

\section{References}

1. Gaziano TA, Pagidipati N. Scaling up chronic disease prevention interventions in lower- and middle-income countries. Апnи Rev Public Health. 2013;34:317-335.

2. Mendis S, Alwan A. Prioritized research agenda for prevention and control of noncommunicable diseases. Geneva: WHO; 2011.

3. Clark AM, Hartling L, Vandermeer B, et al. Metaanalysis: secondary prevention programs for patients with coronary artery disease. Ann Intern Med. 2005;143:659-672.

4. O'Gara PT, Kushner FG, Ascheim DD, et al. 2013 ACCF/AHA guideline for the management of ST-elevation myocardial infarction: a report of the American College of Cardiology Foundation/American Heart Association Task Force on Practice Guidelines. J Circulation. 2013; 127(4):e362-425.

5. Ibanez B, James S, Agewall S, et al. ESC Scientific Document Group. 2017 ESC Guidelines for the management of acute myocardial infarction in patient's presenting with ST-segment elevation: The Task Force for the management of acute myocardial infarction in patients presenting with ST-segment elevation of the European Society of Cardiology (ESC). Eur Heart J. 2018;39(2):119-177.

6. Scott IA, Lindsay KA, Harden HE. Utilisation of outpatient cardiac rehabilitation in Queensland. Med J Aust. 2003;179:341-345.

7. Barber K, Stommel M, Kroll J. Cardiac rehabilitation for communitybased patients with myocardial infarction: factors predicting discharge recommendation and participation. JClin Epidemiol. 2001;54(10):10251030.

8. Neubeck L, Freedman SB, Clark AM, et al. Participating in cardiac rehabilitation: a systematic review and metasynthesis of qualitative data. Eur J Prev Cardiol. 2012;19:494-503.

9. Redfern J, Briffa T, Ellis E, et al. Patient-centered modular secondary prevention following acute coronary syndrome. $J$ Cardiopulm Rehabil Prev. 2008;28:107-115.

10. Thygesen K, Alpert JS, Jaffe AS, et al. Third universal definition of myocardial infarction. Circulation. 2012; 126(16):2020-2035.

11. Appleby MA, Angeja BG, Dauterman K, et al. Angiographic assessment of myocardial perfusion: TIMI myocardial perfusion (TMP) grading system. Heart. 2001;86(5):485-486.

12. Haskell WL, Alderman EL, Fair JM, et al. Effects of intensive multiple risk factor reduction on coronary atherosclerosis and clinicalcardiac events in men and women with coronary artery disease. The 
Stanford Coronary RiskIntervention Project (SCRIP). Circulation. 1994;89(3):975-990.

13. Williams PT. Vigorous exercise, fitness and incident hypertension, high cholesterol, and diabetes. Med Sci Sports Exerc. 2008;40(6):998-1006.

14. Borgundvaag E, Janssen I. Objectively Measured Physical Activity and Mortality Risk Among American Adults. Am J Prev Med. 2017;52(1):e25-e31.

15. Murry CE, Jennings RB, Reimer KA. Preconditioning with ischemia: a delay of lethal cell injury in ischemic myocardium. Circulation. 1986;74:1124-1136.

16. Kendziorra K, Walther C, Foerster M, et al. Changes in myocardial perfusion due to physical exercise in patients with stable coronary artery disease. Eur J Nucl Med Mol Imaging. 2005;32:813-819.

17. Fletcher GF, Ades PA, Kligfield P, et al. Exercise standards for testing and training: a scientific statement from the American Heart Association. Circulation. 2013;128:873-934.

18. Sandesara PB, Lambert CT, Gordon NF, et al. Cardiac rehabilitation and risk reduction: time to "rebrand and reinvigorate". $J$ Am Coll Cardiol. 2015;65(4):389-395.

19. Turk-Adawi KI, Grace SL. Narrative review comparing the benefits of and participation in cardiac rehabilitation in high-,middle- and lowincome countries. Heart Lung Circ. 2015;24(5):510-520.

20. Jolliffe JA, Rees K, Taylor RS, et al. Exercise-based rehabilitation for coronary heart disease. Cochrane Database Syst Rev. 2001;(1):CD001800.

21. Jiang X, Sit JW, Wong TK. A nurse-led cardiac rehabilitation programme improves health behaviours and cardiac physiological risk parameters: Evidence from Chengdu, China. J Clin Nurs. 2007; 16:1886-1897.

22. El Demerdash S, Khorshid H, Salah I, et al. Cardiac rehabilitation improves the ischemic burden in patients with ischemic heart disease who are not suitable for revascularization. Cardiovasc Revasc Med. 2015;16(5):280-283.

23. Lawler PR, Filion KB, Eisenberg MJ. Efficacy of exercisebased cardiac rehabilitation post-myocardial infarction: a systematic reviewand meta-analysis of randomized controlled trials. Am Heart J. 2011;162(4):571-584.

24. Ries AL, Bauldoff GS, Carlin BW. Pulmonary Rehabilitation: Joint ACCP/AACVPR Evidence-Based Clinical Practice Guidelines. Chest. 1997;112(5):1363-1396.

25. Ribeiro F, Oliveira NL, Silva G, et al. Exercise based cardiac rehabilitation increases daily physical activity of patients following myocardial infarction: subanalysis of two randomised controlled trials. Physiotherapy. 2017;103(1):59-65.

26. Snoek JA, Berkel S, Meeteren N, et al. Effect of aerobic training on heart rate recovery in patients with established heart disease; a systematic review. PloS one. 8(12):e83907.

27. Haddadzadeh MH, Maiya AG, Padmakumar R, et al. Effect of exercise-based cardiac rehabilitation on ejection fraction in coronary artery diseasepatients: a randomized controlled trial. Heart Views. 2011;12(2):51-57.

28. Buckingham SA, Taylor RS, Jolly K, et al. Home-based versus centre based cardiac rehabilitation: abridged Cochrane systematic reviewand meta-analysis. Open Heart. 2016;3(2):e000463.

29. Senuzun F, Fadiloglu C, Burke LE, et al. Effects of home-based cardiac exercise program on the exercise tolerance, serum lipid values and self-efficacy of coronary patients. Eur J Cardiovasc Prev Rehabil. 2006;13(4):640-645.

30. Chen JT, Lin TH, Voon WC, et al. Beneficial effects of home-based cardiac rehabilitation on metabolic profiles in coronary heart-disease patients. Kaohsiung J Med Sci. 2016;32(5):267-275.

31. Mosleh SM, Bond CM, Lee AJ, et al. Effects of community based cardiac rehabilitation: Comparison with a hospital-based programme. Eur J Cardiovasc Nurs. 2015;14(2):108-116.

32. Dalal HM, Evans PH, Campbell JL, et al. Home-based versus hospital based rehabilitation after myocardial infarction: A randomized trialwith preference arms--Cornwall Heart Attack Rehabilitation Management Study (CHARMS). Int J Cardiol. 2007;119(2):202-211.

33. Bravo-Escobar R, González-Represas A, Gómez-González AM, et al. Effectiveness and safety of a home-based cardiac rehabilitation programme of mixed surveillance in patients with ischemic heart disease at moderate cardiovascular risk: A randomised, controlled clinical trial. BMC Cardiovasc Disord. 2017;17(1):66.

34. Shagufta S, Moiz J A, Aggarwal R. Effect of Supervised Versus Home Based Cardiac Rehabilitation on Heart Rate Recovery in Patients with Coronary Artery bypass Grafting Design Setting. Indian J Physiother Occup Ther. 2011;5(3):199-202.

35. Morshedi-Meibodi A, Larson MG, Levy D, et al. Heart rate recovery after treadmill exercise testing and risk of cardiovascular disease events (The Framingham Heart Study). Am J Cardiol. 2002;90(8):848-852.

36. Xu L, Cai Z, Xiong M, et al. Efficacy of an early home based cardiac rehabilitation program for patients after acute myocardial infarction: A three-dimensional speckle tracking echocardiography randomized trial. Medicine (Baltimore). 2016;95(52):e5638. 